\title{
Archéopages
}

Archéopages

Archéologie et société

Hors-série 1 | 2008

Construction $^{s}$ de l'archéologie

\section{Des tranchées aux tranchées : les méandres de l'archéologie « gauloise » dans l'Aisne}

\section{Sylvain Thouvenot et Sophie Desenne}

\section{(2) OpenEdition}

1 Journals

Édition électronique

URL : https://journals.openedition.org/archeopages/855

DOI : 10.4000/archeopages.855

ISSN : 2269-9872

Éditeur

INRAP - Institut national de recherches archéologiques préventives

Édition imprimée

Date de publication : 1 février 2008

Pagination : 70-74

ISSN : 1622-8545

\section{Référence électronique}

Sylvain Thouvenot et Sophie Desenne, « Des tranchées aux tranchées : les méandres de l'archéologie " gauloise » dans l'Aisne », Archéopages [En ligne], Hors-série 1 | 2008, mis en ligne le 01 février 2008, consulté le 24 février 2023. URL : http://journals.openedition.org/archeopages/855 ; DOI : https:// doi.org/10.4000/archeopages.855 

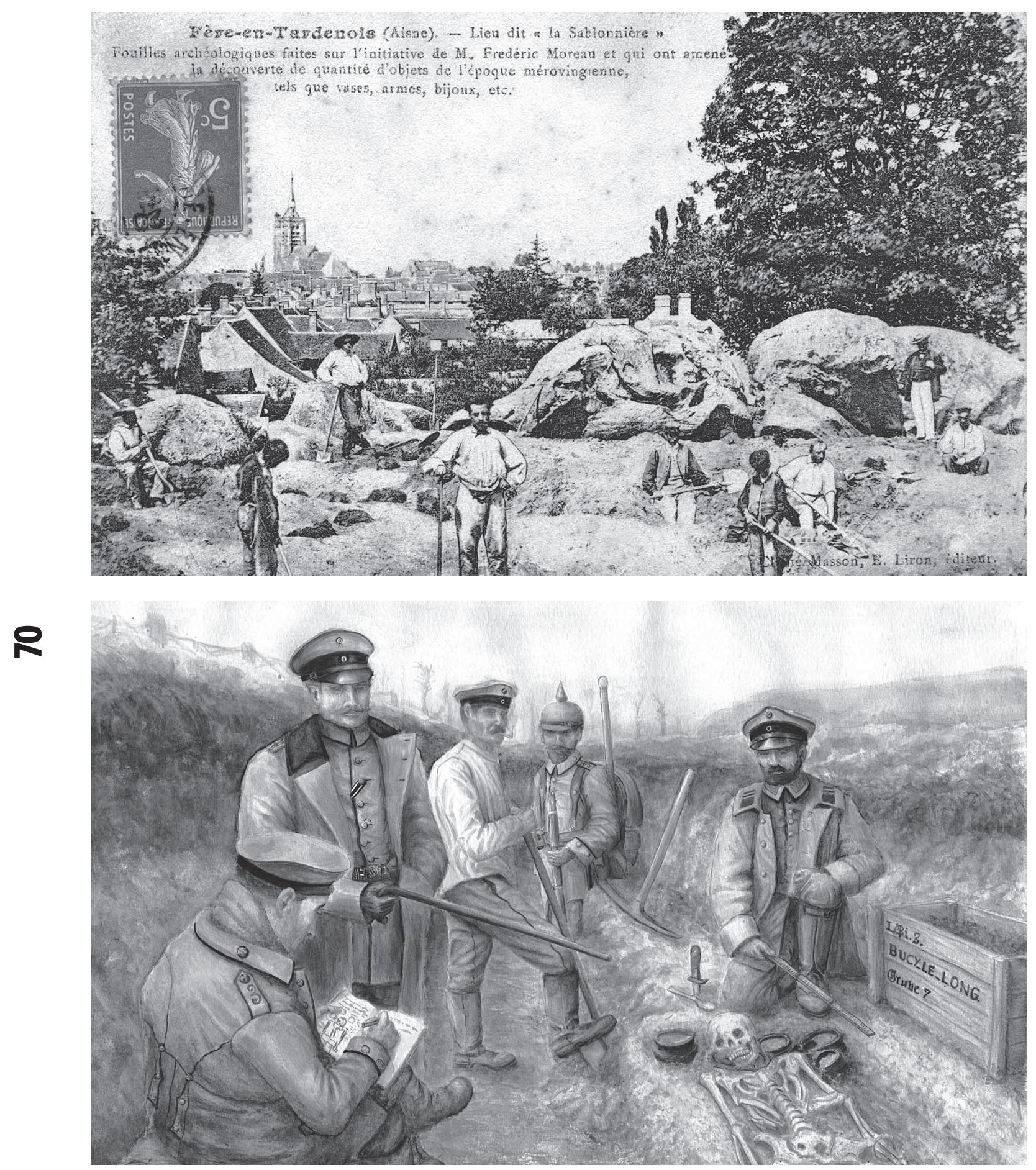

[Fig. 1] Fouilles d'un cimetière

à Fère-en-Tardenois «Sablonnières" (Aisne) par F.Moreau en 1875-1878. Carte postale vers 1910.
[Fig.2] Fouilles de la nécropole celtique de Bucy-le-Long «la Héronnière» (Aisne) par l'armée allemande en 1915. 


\section{Des tranchées aux tranchées : les méandres de l'archéologie «gauloise» dans l'Aisne}

\author{
Sylvain Thouvenot \\ Sophie Desenne \\ Inrap
}

$\mathrm{L}$

e temps est à l'urgence! Urgence pour

l'archéologie de libérer le terrain, d'analyser,

de synthétiser, de communiquer, de s'adapter au rythme de la société pour exister. En somme, rien de nouveau! Dans l'histoire contemporaine, la recherche archéologique en tant que science humaine a toujours été l'objet d'enjeux culturels, sociaux et politiques qui ont encouragé ou entravé sa propre évolution. Sur ce point, la chronique de la seule archéologie de lâge du Fer, vue à un échelon local, est riche d'éclairages et d'enseignements quant aux méandres de l'histoire de notre discipline.

Dans l'Aisne, la première mention de sépultures gauloises remonte à 1767 . Brayer, avocat au bailliage de Soissons, informe un journal parisien de la découverte d'une dizaine de tombes avec mobilier dans les environs de Soissons. Malgré ce premier allant de curiosité, il faut attendre une centaine d'années pour qu'émerge une archéologie de la période gauloise dans ce département. Qu'est-il advenu, en un siècle et demi d'existence, tant des données accumulées que du patrimoine archéologique?

1850-1914 - Les tranchées des pionniers de l'archéologie axonaise. Comme dans de nombreuses régions, le milieu du XIX ${ }^{\mathrm{e}}$ siècle marque, dans l'Aisne, la naissance de l'archéologie. La création par Napoléon III d'une archéologie de la Gaule constitue alors le terreau d'une identité nationale instrumentalisée. Elle trouve localement écho dans les sociétés savantes qui existent dans pratiquement chaque ville. À partir des années 1870 et jusqu'à la fin du siècle, cet engouement attire des notables philanthropes et collectionneurs (A. Nicaise, F.Moreau, E. Piette, J.Pilloy...), et autant de pilleurs (dont le fameux Le Laurain). Si la Marne devient une terre «gauloise», l'Aisne semble plutôt «franque», combien même la somme des tombes laténiennes mises au jour excède plusieurs milliers. L'intérêt se porte aussi sur les sites fortifiés gaulois de la conquête romaine, cités par César. Ces fouilles par tranchées exploratoires manuelles se font au gré de leur inventeur [Fig.1]. Cependant, certains savants mettent scientifiquement à profit les découvertes fortuites occasionnées par les grands travaux d'aménagement (canaux, chemin de fer, carrières de granulats...).

En ces temps pionniers, la recherche archéologique fut souvent consciencieuse et documentée, mais elle n'évita ni la dispersion des collections entre musées et fonds privés ni le pillage. Certes, les quelques sources documentaires existantes sont inégales, mais on se doit de saluer aujourd'hui l'initiative de certains, qui ont vendu ou légué leurs collections et leur documentation au domaine public. Nombreux sont les archéologues actuels qui ont pu profiter de la collection Moreau au Musée d'archéologie nationale ou des fonds préservés dans divers musées.

1914-1918 - Des pioniers en première ligne dans les tranchées. La Grande Guerre est le temps de la rupture et du paroxysme pour l'archéologie dans l'Aisne. À côté des destructions massives qui ont affecté le patrimoine bâti, mobilier et enfoui, elle emporte avec elle la plus grande part des collections privées et des musées locaux, autant que les archéologues eux-mêmes, tel J.Déchelette, mort au champ d'honneur près de Soissons.

Du chaos, l'archéologie tire aussi parfois son parti. En janvier 1915, du côté français, alors que H.Barbusse relate dans Le Feu l'anecdotique découverte d'un outil en silex dans une tranchée du front de l'Aisne, la priorité est ailleurs. À l'inverse, à quelques centaines de mètres de là, des soldats allemands du génie, des pioniere, s'apprêtent quant à eux, sur ordre de leur plus haute hiérarchie, à entamer des fouilles méthodiques sur la nécropole de Bucy-le-Long qu'a révélée le creusement de leurs tranchées [Fig.2]. Si la réalisation de cette fouille et la transmission de la documentation et des objets à un musée berlinois ne prennent leur importance que quatre-vingt-dix ans plus tard avec l'étude complète du site, elles illustrent bien l'usage officiel qui fut fait alors de la discipline à des fins de propagande identitaire. De fait, le cas n'est pas isolé. Plusieurs fouilles programmées de palais carolingiens et d'un cimetière mérovingien axonais n'auront d'autre but que de justifier l'occupation allemande sur des territoires «germanisés» de longue date.

1918-1960 - Où l'on inventorie les sites détruits. La Première Guerre mondiale engendre une seconde phase, tout aussi dommageable pour les archives du sol: la nécessaire reconstruction des «Régions libérées». L'urgence patrimoniale est à la sauvegarde et à la restauration du bâti. L'archéologie locale va alors entrer dans un long sommeil où les bonnes volontés n'auront d'autre solution que de recenser les découvertes fortuites occasionnées par des travaux de tous ordres. À peine remise du premier conflit, l'Aisne subira de plein fouet la Seconde Guerre mondiale. C'est donc au plus creux de la vague que l'archéologie trouvera un ancrage durable avec la promulgation de la loi de 1941. Sa portée n'aura toutefois aucune influence en «zone occupée». Malgré l'instauration des directions des Antiquités en 1945, la loi n'influera guère sur les conséquences archéologiquement destructives des «trente glorieuses» débutantes: industrialisation, développement urbain, mécanisation de l'agriculture... 


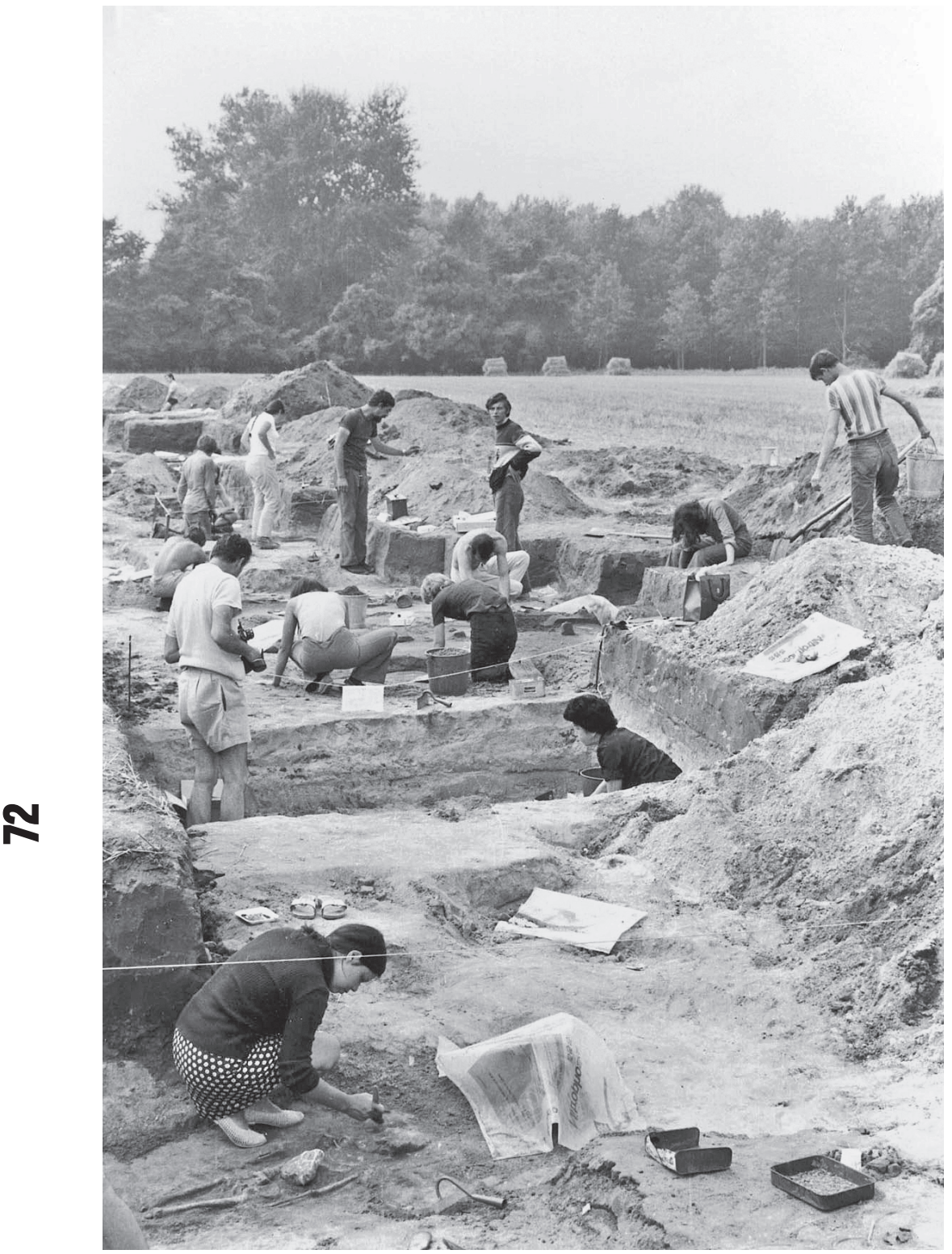

[Fig.3] Fouilles de la nécropole celtique de Bucy-le-Long «la Héronnière» (Aisne) par G.Lobjois en 1971-1977 
1960-1972 - Les bénévoles font front: les premières fouilles de sauvetage. Alors que la construction bat son plein et que les villes s'étendent, le besoin de granulats favorise l'ouverture de nombreuses sablières, notamment dans les vallées alluviales. À la faveur d'un contexte social, intellectuel et réglementaire favorable (décret de 1964), ces travaux de plus en plus mécanisés et consommateurs de grandes surfaces suscitent la réaction et l'intervention d'archéologues bénévoles, souvent correspondants locaux des services de l'État, afin de sauver les nombreux sites menacés. Faute de réels moyens, aidés par des bénévoles en vacances, leur capacité de pression auprès des aménageurs est souvent limitée. Pour cette période, la contribution de B.Ancien, M.Boureux, J.Debord ou G.Lobjois, parmi d'autres, a été essentielle à la naissance d'une archéologie de sauvetage dans l'Aisne. On leur doit des études majeures sur des sites comme l'oppidum de Villeneuve-Saint-Germain ou les nécropoles de Pernant et de Bucy-le-Long [Fig.3]. Leur motivation étant purement scientifique, l'intégralité des données est allée au domaine public.

1972-1985 - Le temps des « Parisiens » ou la naissance d'un programme de recherche. Si la crise de 1973 représente un frein au développement économique, cette période correspond pourtant à une véritable transformation de l'archéologie locale. Des «Parisiens», enseignants, chercheurs et étudiants, découvrent la vallée de l'Aisne et ses villages néolithiques menacés [Fig.4]. La pertinence d'un développement de la recherche archéologique dans un cadre préventif, à l'image des expériences d'Europe centrale, convainc ces professionnels d'établir un programme de recherche. Celui-ci a pour objectif l'étude de l'occupation humaine durant la Protohistoire, à partir du suivi systématique des carrières. Il va rapidement prendre de l'ampleur du fait d'un réseau dynamique d'archéologues tant professionnels que bénévoles, d'un riche potentiel patrimonial, d'une sensibilisation croissante des acteurs économiques et d'une implication des instances archéologiques institutionnelles (ministère de la Culture, CNRS et Université) auxquelles s'ajoute souvent le soutien logistique de la population et des collectivités locales. C'est le temps des grands décapages désormais mécanisés sur plusieurs dizaines d'hectares annuels et l'amorce d'un financement de l'archéologie de sauvetage. Cette dynamique d'équipe contribuera à l'exploration de nombreux sites de l'âge du Fer et à une véritable émulation de la recherche.

1986-2001 - Les professionnels entrent dans la carrière. Dans l'Aisne, l'année 1986 marquera l'extension des interventions à d'autres aménagements, en plus du programme en carrières existant: tracé autoroutier $\mathrm{A} 26$ et oppidum de Condé-sur-Suippes, Variscourt.
Elle marque aussi le début d'une professionnalisation accrue des membres de l'équipe à l'Afan et d'une mutualisation des moyens, dans l'attente d'un cadre législatif rénové.

Les sauvetages au coup par coup se transforment en archéologie préventive systématique et le champ d'étude concerne désormais l'intégralité des vestiges, situation dont bénéficient évidemment ceux de La Tène. Grâce aux grandes surfaces explorées, les petites implantations et les grands sites sont traités de manière égale selon un protocole éprouvé mais ayant du mal à intégrer la masse documentaire grandissante. C'est le temps de l' «archéologie en caisses»!

2001-2007 - Le retour aux tranchées.

La mise en application du nouveau cadre législatif et la création de l'Inrap et d'autres opérateurs concrétisent une longue évolution de l'archéologie préventive, ici comme sur l'ensemble du territoire national. L'activité devient totalement professionnelle, les méthodes sont rodées et les interventions généralisées à tous les types d'aménagement. Dans l'Aisne, Zac, tracés linéaires et lotissements prennent le pas sur les carrières. Désormais, le diagnostic préalable, par échantillonnage raisonné en tranchées, préside au traitement des vestiges: fouille souvent partielle, destruction raisonnée ou préservation.

L'heure est donc aux choix. Cependant, beaucoup s'interrogent sur les conséquences à terme de la réduction du champ des investigations: comment intégrer les nombreuses implantations humaines aux traces fugaces, notamment celles de la période gauloise. Ce temps est aussi celui d'une mutation de la recherche régionale...

Conclusion. Fluctuant, parfois chaotique, l'essor de l'archéologie «gauloise» dans l'Aisne est emblématique de l'histoire de toute la discipline. D'abord philanthropique, elle est devenue peu à peu une activité professionnelle, admise et reconnue par une société qui lui accorde désormais le statut de science humaine à part entière. Cette évolution n'a certes pas été linéaire. Entre les temps de croissance ou de stagnation se sont intercalés des temps de crise, des temps de guerre où l'archéologie a été convoquée comme outil politique et identitaire. Durant toutes ses étapes de maturation où l'altération du patrimoine enfoui n'a cessé de croître, la réaction collective aux destructions n'a vraiment abouti que sous l'impulsion d'individus passionnés et convaincus.

Cette petite chronique nous permet également de constater que, si bien fouiller sauve le passé, une bonne conservation des archives et du mobilier par la collectivité assure la pérennité des connaissances. 


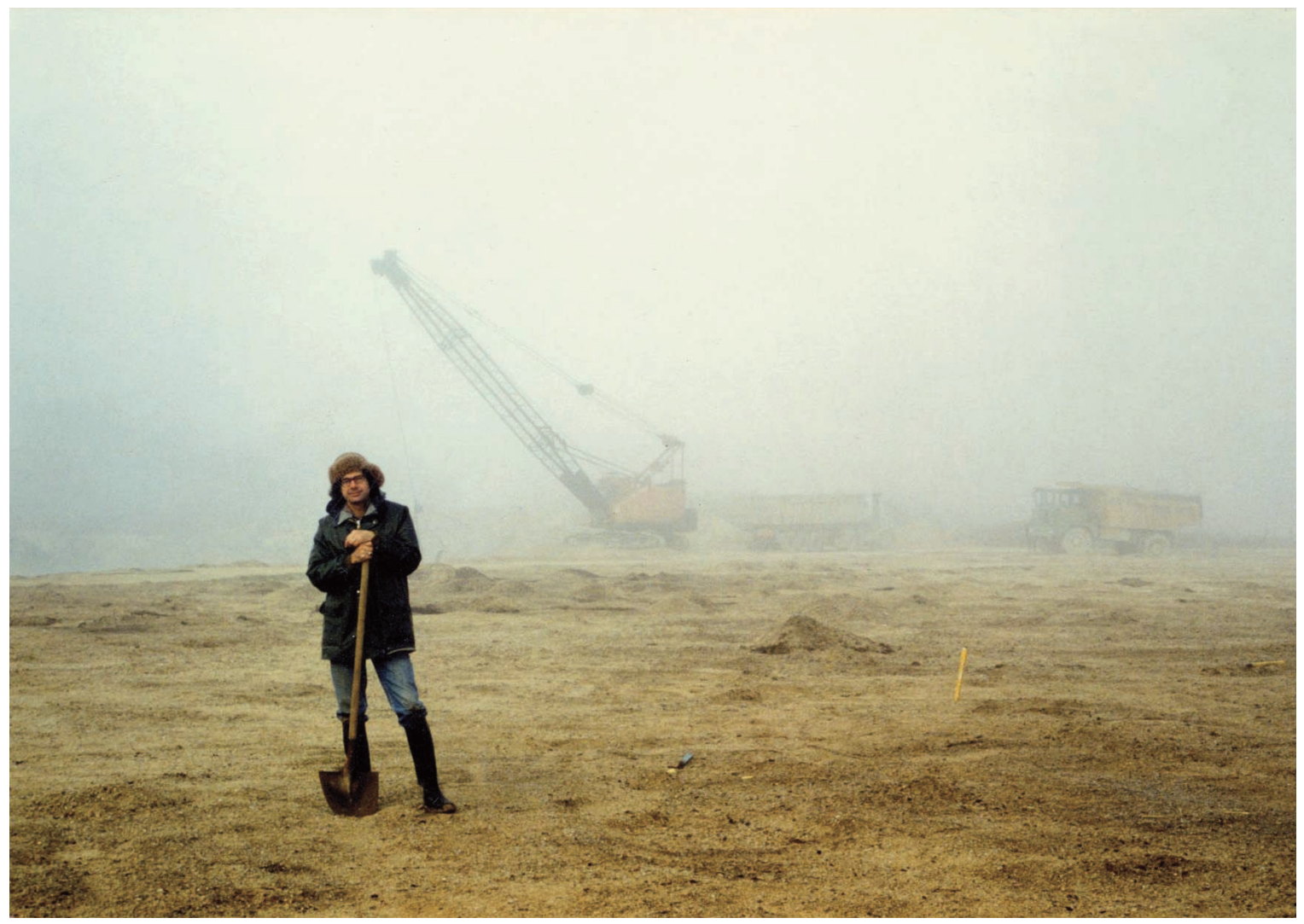

[Fig.4] Suivi d'un décapage extensif dans la carrière de Berry-au-Bac «le Vieux-Tordoir» (Aisne)

par un enseignant-chercheur dans les années 1980. 